\title{
Impact of acute stress on psychomotor bimanual performance during a simulated tumor resection task
}

\author{
Khalid Bajunaid, MD, MSc, ${ }^{1,2}$ Muhammad Abu Shadeque Mullah, MSc, ${ }^{3}$ \\ Alexander Winkler-Schwartz, MD, ${ }^{1}$ Fahad E. Alotaibi, MD, MSc, ${ }^{1,5}$ Jawad Fares, BSc, ${ }^{1}$ \\ Marta Baggiani, ${ }^{1}$ Hamed Azarnoush, PhD, ${ }^{1,7}$ Sommer Christie, BSc, MHK, ${ }^{4}$ \\ Gmaan Al-Zhrani, MD, MA, ${ }^{1,5}$ Ibrahim Marwa, MD,1 \\ Abdulrahman Jafar Sabbagh, MBChB, FRCSC, ${ }^{1,5,6}$ Penny Werthner, PhD, ${ }^{4}$ and \\ Rolando F. Del Maestro, MD, PhD'
}

${ }^{1}$ Neurosurgical Simulation Research and Training Center, Department of Neurosurgery, Montreal Neurological Institute and Hospital; ${ }^{3}$ Department of Epidemiology, Biostatistics and Occupational Health, McGill University, Montreal, Quebec; ${ }^{4}$ University of Calgary, Faculty of Kinesiology, Calgary, Alberta, Canada; 'Division of Neurosurgery, Faculty of Medicine, University of Jeddah, Jeddah; ${ }^{5}$ National Neuroscience Institute, Department of Neurosurgery, King Fahad Medical City; ${ }^{6}$ Faculty of Medicine, King Saud bin Abdulaziz University for Health Sciences, Riyadh, Saudi Arabia; and 'Department of Biomedical Engineering, Tehran Polytechnic, Tehran, Iran

OBJECTIVE Severe bleeding during neurosurgical operations can result in acute stress affecting the bimanual psychomotor performance of the operator, leading to surgical error and an adverse patient outcome. Objective methods to assess the influence of acute stress on neurosurgical bimanual psychomotor performance have not been developed. Virtual reality simulators, such as NeuroTouch, allow the testing of acute stress on psychomotor performance in risk-free environments. Thus, the purpose of this study was to explore the impact of a simulated stressful virtual reality tumor resection scenario by utilizing NeuroTouch to answer 2 questions: 1) What is the impact of acute stress on bimanual psychomotor performance during the resection of simulated tumors? 2) Does acute stress influence bimanual psychomotor performance immediately following the stressful episode?

METHODS Study participants included 6 neurosurgeons, 6 senior and 6 junior neurosurgical residents, and 6 medical students. Participants resected a total of 6 simulated tumors, 1 of which (Tumor 4 ) involved uncontrollable "intraoperative" bleeding resulting in simulated cardiac arrest and thus providing the acute stress scenario. Tier 1 metrics included extent of blood loss, percentage of tumor resected, and "normal" brain tissue volume removed. Tier 2 metrics included simulated suction device (sucker) and ultrasonic aspirator total tip path length, as well as the sum and maximum forces applied in using these instruments. Advanced Tier 2 metrics included efficiency index, coordination index, ultrasonic aspirator path length index, and ultrasonic aspirator bimanual forces ratio. All metrics were assessed before, during, and after the stressful scenario.

RESULTS The stress scenario caused expected significant increases in blood loss in all participant groups. Extent of tumor resected and brain volume removed decreased in the junior resident and medical student groups. Sucker total tip path length increased in the neurosurgeon group, whereas sucker forces increased in the senior resident group. Psychomotor performance on advanced Tier 2 metrics was altered during the stress scenario in all participant groups. Performance on all advanced Tier 2 metrics returned to pre-stress levels in the post-stress scenario tumor resections.

CONCLUSIONS Results demonstrated that acute stress initiated by simulated severe intraoperative bleeding significantly decreases bimanual psychomotor performance during the acute stressful episode. The simulated intraoperative bleeding event had no significant influence on the advanced Tier 2 metrics monitored during the immediate post-stress operative performance.

http://thejns.org/doi/abs/10.3171/2015.5.JNS15558

KEY WORDS acute stress; uncontrolled bleeding; brain tumor; NeuroTouch; virtual reality simulator; performance metrics; psychomotor performance; surgical technique 
$\mathrm{S}$ URGEONS consider severe, difficult to control, intraoperative bleeding as one of the most common causes of acute stress in the operating room. ${ }^{4,5,34}$ Acute stress can be defined as a "relationship with the environment that the person appraises as significant for his or her well-being and in which the demands tax or exceed available coping resources." 22 Acute stress can be real (threat to personal well-being) or implied (self-doubt, fear of failure). And although surgeons are not in immediate personal danger in a stressful situation in the operating room, they do exhibit similar physiological and psychological reactions to a perceived significant risk to the patient. ${ }^{25}$ During neurosurgical tumor operations, significant bleeding can occur, and occasionally this blood loss can result in bradycardia and cardiac arrest. Accurately measuring the range of psychomotor skills employed by the "expert" in the operating room when faced with acute stress-inducing events and his or her skills after experiencing such stress is difficult. ${ }^{16}$ In the military and aviation industries, medicine, and high-performance sports, acute stress has been recognized as a significant factor affecting performance..$^{11,17,30}$ These fields have implemented training methods and strategies to improve performance and coping mechanisms. ${ }^{17,26,27,30}$ The ability to objectively measure a neurosurgeon's technical skills during and after acute stress-inducing bleeding events in the operating room may lead to a better understanding of how to mitigate the adverse effects of stress on neurosurgical performance. Using the NeuroTouch virtual reality simulator, we have developed and validated a series of technical skills assessment tools that can objectively measure bimanual psychomotor performance skills. ${ }^{1-3,8,10,12,15,16}$ This platform allowed the development of simulated scenarios in which we could assess the effect of acute stress secondary to severe bleeding during brain tumor resection on bimanual psychomotor performance before, during, and immediately following the stress-inducing event. ${ }^{1,2,8}$

This study was designed to answer 2 questions: 1) What is the impact of acute stress on bimanual psychomotor performance during the resection of simulated tumors? 2) Does acute stress influence bimanual psychomotor performance immediately following the stressful episode? The answers to these questions allowed us to begin developing a framework outlining the specific psychomotor skills that experts use during stress-inducing events associated with significant bleeding.

\section{Methods}

McGill University Institutional Review Board approved the study protocol, and consent was signed by all study participants including 24 individuals -6 boardcertified neurosurgeons, 6 senior (PGY4-6) and 6 junior neurosurgical residents (PGY1-3), and 6 senior medical students from the same institute. Collected demographic data included age, sex, handedness, resident training level, and hours of video games and musical instruments played weekly.

\section{State-Trait Anxiety Inventory}

Stress perception was assessed using the validated short form of the State-Trait Anxiety Inventory (STAI) questionnaire adapted to this study. ${ }^{7,24}$ The questionnaire was completed immediately before and after completing the simulated tasks. The STAI questionnaire consisted of 6 items (calm, tense, upset, relaxed, content, and worried), as adapted for surgical trials, measured on a 4-point Likert scale (Table 1). The minimum achievable STAI score was 6 and the maximum was 24, where higher scores imply greater anxiety perception.

\section{Simulated Virtual Reality Scenarios}

To address the study questions, we developed 3 simulated brain tumor scenarios involving 2 tumors each (6 tumors total) with an identical visual appearance, stiffness, and random bleeding points. ${ }^{1,8,12}$ The color and circular structure chosen for each tumor was a simulated glioma-like brain tumor (Fig. 1A). ${ }^{1}$ Each tumor was embedded in a simulated cortical surface (Young's modulus 3 $\mathrm{kPa}$ ) differentiated from a white matter-like background (Young's modulus $3 \mathrm{kPa}$ ). This provided a distinct border interface between the tumor (Young's modulus $9 \mathrm{kPa}$ ) and surrounding structures (Young's modulus $3 \mathrm{kPa}$ ). To accurately represent the range of human brain and tumor stiffness, a tactile cue in our scenarios, we assessed multiple samples from 11 different human glial tumors (Young's modulus). ${ }^{8,10,12}$

In Scenario 1 both tumors (Tumors 1 and 2) were identical, and during all resections an initial heart rate of 60 bpm was heard. The first tumor (Tumor 3) in Scenario 2 was similar to those in Scenario 1, but the second tumor (Tumor 4) was designed as the stress scenario in which rapid and uncontrollable bleeding occurred immediately on attempted tumor removal using the simulated aspirator and/or suction device (sucker). At 75 seconds into this tumor resection, the audible heart rate dropped to $30 \mathrm{bpm}$ at 90 seconds and $15 \mathrm{bpm}$ at 105 seconds, and cardiac arrest was simulated for the remaining 15 seconds of the scenario. Immediately following this event, participants were asked to perform resections in 2 further scenarios in which the tumors (Tumor 5 and 6) were identical to those in the first 3, allowing assessment of bimanual psychomotor performance following an acute stress event. A video demonstration of the task is featured in Video 1.

VIDEO 1. Video demonstration of Scenario 2: resection of Tumors 3 and 4. Copyright Khalid Bajunaid. Published with permission. Click here to view.

\section{Simulated Operative Resection Procedures}

This study was conducted utilizing the previously described NeuroTouch platform (Fig. 1C). ${ }^{1-3,8,10,12,15,16,18,31,32}$ All operators were acquainted with the system since they had enrolled in a previous trial involving the resection of simulated tumors. ${ }^{3}$ None of the participants knew that the purpose of the study was to assess the influence of acute stress and the sequence of the scenarios or had heard information related to this trial. Two individuals with foreknowledge of the trial's intention were excluded. Individuals who finished the trial were asked not to relate trial information with any possible participants. The goal, defined verbally and in writing, was the resection of 6 simu- 
TABLE 1. Short form of the STAI questionnaire

\begin{tabular}{ccccc}
\hline & \multicolumn{4}{c}{ Response Scores } \\
\cline { 2 - 5 } Statement & Not at All & Somewhat & Moderately So & Very Much \\
\hline I feel calm & 1 & 2 & 3 & 4 \\
\hline I feel tense & 1 & 2 & 3 & 4 \\
\hline I feel upset & 1 & 2 & 3 & 4 \\
\hline I am relaxed & 1 & 2 & 3 & 4 \\
\hline I am content & 1 & 2 & 3 & 4 \\
\hline I am worried & 1 & 2 & 3 & 4 \\
\hline
\end{tabular}

lated brain tumors with minimal removal of background tissue representing simulated "normal" brain tissue. The procedure was accomplished using a bimanual technique with a simulated suction device to control bleeding sites in the nondominant hand and a simulated ultrasonic aspirator for tumor removal in the dominant hand (Fig. 1B and $\mathrm{C}$ ). The simulated sucker and aspirator were set at functional levels that resulted in adequate bleeding control and tumor removal as determined in previous studies and could not be modified. Using a predefined sequence, participants began by resecting the left tumor followed by resection of the right in Scenario 1 and followed this sequence for Scenarios 2 and 3. In previous studies 3 minutes was allowed for complete resection of a simulated tumor of this shape and size. ${ }^{1,3,8}$ In the present study, however, only 2 minutes was allocated to resect each tumor to further increase stress. Participants could not revisit the left tumor once they commenced resection of the right. ${ }^{1,3,8}$

\section{NeuroTouch Metrics}

Safety, quality, and efficiency of simulated operative procedures can be assessed utilizing Tier 1 and Tier 2 metrics as we have previously described. ${ }^{1-3,8}$ Independent hand motor skills were measured using Tier 2, and advanced Tier 2 metrics were specifically designed and validated to assess motor and cognitive neurosurgical bimanual skills interaction. ${ }^{1,2}$ Assessed Tier 1 metrics included amount of blood loss, tumor percentage resected, and brain volume removed. Tier 2 metrics included instrument total tip path length, maximum force applied, and sum of forces used. Advanced Tier 2 metrics assessed were efficiency index, coordination index, ultrasonic aspirator path length index, and ultrasonic aspirator bimanual forces ratio.

\section{Psychophysiological Assessment}

The operative and resident training environment can be extremely stressful, resulting in both physiological and cognitive alterations. ${ }^{9,23}$ Physiological (heart rate, respiration rate, heart rate variability, electromyographic activity, and peripheral body temperature) and neurological (electroencephalographic activity) responses were measured at baseline, during the resection of each tumor, and during rest periods between each scenario. ${ }^{13,28,33}$ These studies will be the focus of further communications.

\section{Statistical Analysis}

Demographics and STAI data are summarized in terms of the mean \pm standard deviation. Since we assessed 24 participants and each participant performed several op-
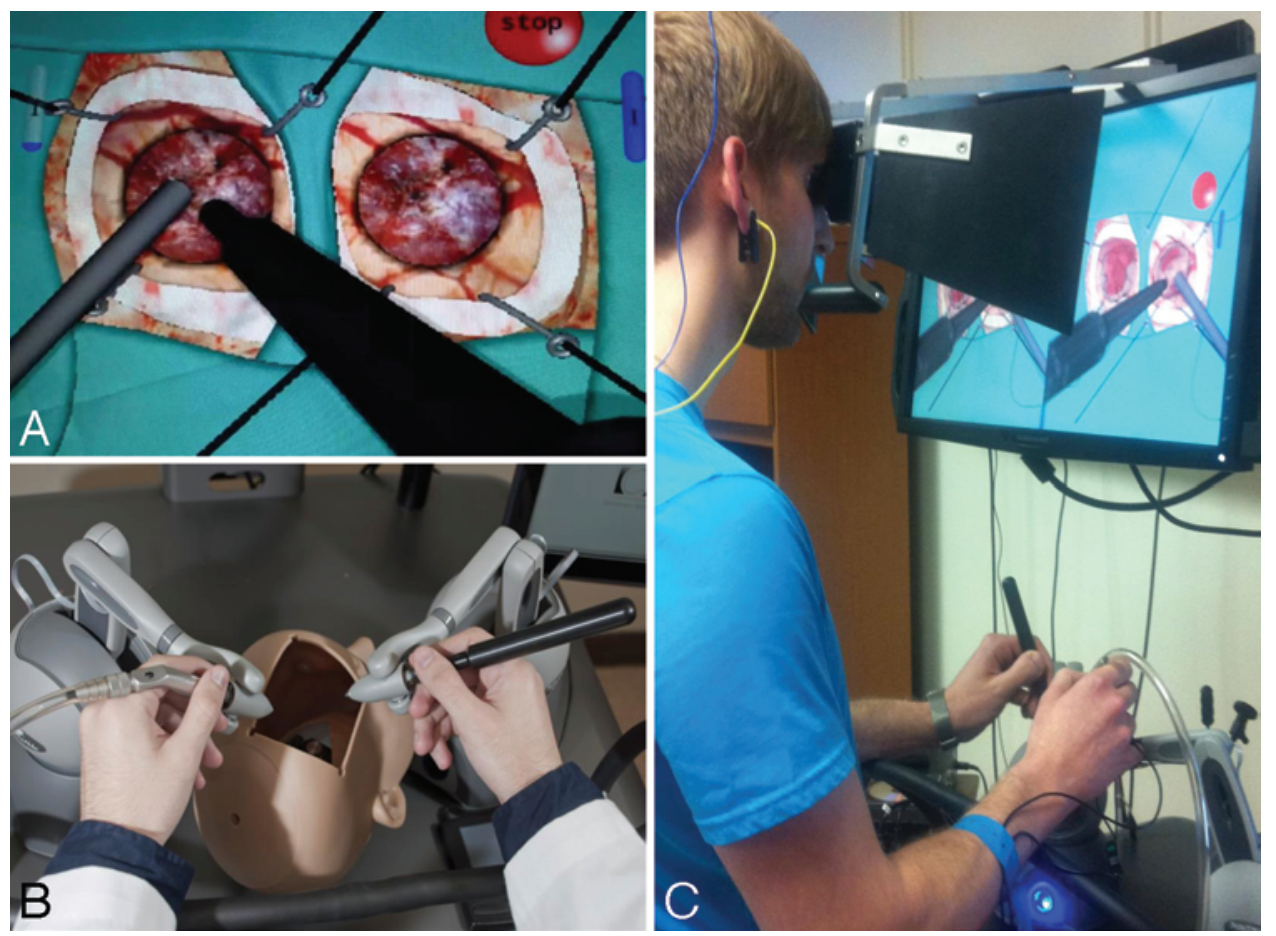

FIG. 1. A: View of the operating scene. B: Mannequin head with 2 haptic devices that provide force feedback for the simulated suction device and ultrasonic aspirator. C: Photograph of a left-handed operator using NeuroTouch with a simulated ultrasonic aspirator in his left hand and sucker in his right, as well as the physiological monitors used during this study. Figure is available in color online only. 
erations on similar types of tumor, the repeated outcomes from each subject were considered to be correlated. ${ }^{1}$ These correlated outcomes were analyzed using linear mixedeffects models. ${ }^{20} \mathrm{We}$ had previously used such models for similar studies. ${ }^{1}$ Outcome variables were transformed appropriately (for example, taking the log, square root, and so forth) to satisfy the normality assumption of the residuals for fitted models. Results were considered statistically significant at a $\mathrm{p}$ value $<0.05$.

\section{Results}

\section{Demographics and STAI}

The mean age of neurosurgeons was $42.2 \pm 7.3$ years compared with $31.5 \pm 2.1$ years for senior residents, 28.5 \pm 1.9 years for junior residents, and $24.8 \pm 3.6$ years for medical students. Males accounted for $83.3 \%$ of the participants, and $83.3 \%$ of the participants were right handed. Among the 4 groups, 3 participants (12.5\%) played musical instruments, whereas 8 (33\%) reported playing video games. Average sleep time on the night before participating in the study was $5.9 \pm 1.3$ hours, suggesting participants were relatively well rested.

Participants showed a statistically significant increase in mean STAI score when baseline (10.2) and post-scenario values (12.4) were compared. Neurosurgeons demonstrated the highest mean scores (12.5) on the baseline STAI questionnaire; however, the only statistically significant higher baseline values were seen between the neurosurgeon and student (8.3) groups. Statistically significant increases in the post-scenario stress-perception scores were seen only in the junior resident and student groups (Table 2).

\section{Acute Stress and Neurosurgical Performance}

To assess each of our objectives, different linear mixedeffects models were used, 1 per metric. First tumor results were not included in our data since the exercise was considered a rehearsal scenario allowing participants to reacquaint themselves with the system. Measurements from Tumors 2 and 3 were combined to provide baseline (pretest) performance values before the stress-inducing Tumor 4 scenario, and those from Tumor 5 and 6 were combined to assess immediate post-stress operative performance.

Baseline blood loss was significantly lower and baseline tumor percentage resected was significantly higher in the neurosurgeon group than in other groups (Fig. 2A). Although neurosurgeons had higher mean values of brain

TABLE 2. State-Trait Anxiety Inventory scores for each participant group pre- and post-scenario*

\begin{tabular}{lcc}
\hline \multicolumn{1}{c}{ Group } & Pre-Scenario & Post-Scenario \\
\hline Staff neurosurgeon & $12.5 \pm 4.6$ & $13.5 \pm 2.1$ \\
\hline Senior resident & $10 \pm 2.3$ & $10.8 \pm 3.9$ \\
\hline Junior resident & $10 \pm 2.7$ & $13.6 \pm 3.1 \dagger$ \\
\hline Senior medical student & $8.3 \pm 1.9$ & $11.5 \pm 5.1 \dagger$ \\
\hline Overall & 10.2 & $12.4 \dagger$ \\
\hline
\end{tabular}

* Results expressed as the means \pm standard deviation.

$\dagger$ Statistically significant $(p<0.05)$. volume removed, these values were only significantly higher than those in the student group. As expected during the stress scenario, a significant increase in blood loss was seen in all groups (Fig. 2B). Tumor percentage resected and brain volume removed decreased significantly during the stress scenario in the junior resident and student groups (Fig. 2C and D).

Baseline Tier 2 values for total tip path length and sum and maximum forces used for both instruments were not statistically different among groups. Neurosurgeons had a statistically significant increase in sucker total tip path length during the stress scenario, consistent with the concept that they were moving the sucker more vigorously while trying to control bleeding (Fig. 3A); however, this increase was not associated with a statistical change in sucker sum or maximum forces used. Senior residents did not significantly increase the sucker total tip path length, but they did significantly increase the sum and maximum forces used with this instrument. Junior residents significantly decreased aspirator total tip path length, and both junior residents and students significantly decreased the aspirator sum forces used during the stress scenario. This result was consistent with their removal of the aspirator from the simulated operative field.

A significant drop in efficiency index, a measure of cognitive-motor skills interaction, calculated as the percentage of time spent actively resecting each tumor divided by the total time allowed for the task, was noted during the stressful scenario in all groups (Fig. 4A). Baseline efficiency index was significantly higher in the neurosurgeon group than in all other groups (Fig. 5). Baseline suction coordination index, a measure of the coordinated use of both instruments simultaneously, was also significantly higher in the neurosurgeon group than in the other groups (Fig. 5). Suction coordination index declined during the stressful scenario among all participant groups and was a statistically significant decline in the neurosurgeon, junior resident, and student groups (Fig. 4C). Baseline ultrasonic aspirator path length index, the percentage of ultrasonic aspirator total tip path length interacting directly with the tumor divided by the overall ultrasonic aspirator total tip path length, was significantly higher in the neurosurgeon group compared with this value in the junior resident and student groups (Fig. 5). Neurosurgeons showed no significant change in this index during the stress scenario, while significant declines were seen in the senior, junior resident, and student groups (Fig. 4B). No significantly different baseline ultrasonic aspirator bimanual force ratio, the average forces applied by the ultrasonic aspirator and sucker divided by the average forces applied by the aspirator alone (a quality of 2-hand interaction), were seen (Fig. 5). Neurosurgeons and senior residents significantly increased their bimanual force ratios during the stress scenario (Fig. 4D). This result is consistent with the concept that they were actively using both instruments to control bleeding.

\section{Psychomotor Performance Immediately Following a Stress-Inducing Event}

For the neurosurgeon group the amount of blood loss after the stress-inducing event is the only metric in which 
A

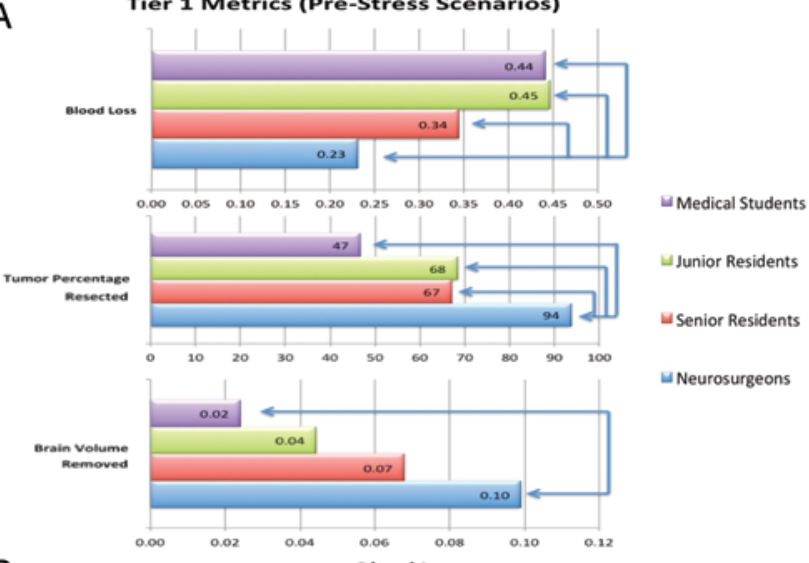

B

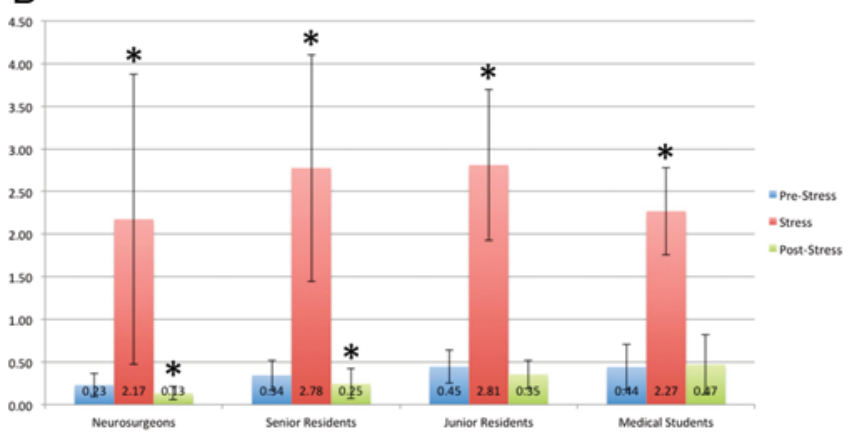

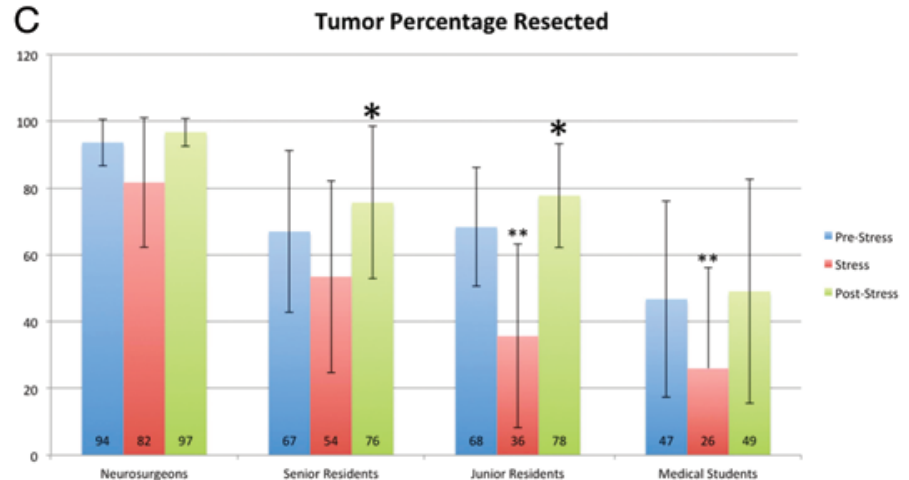

D Brain Volume Removed

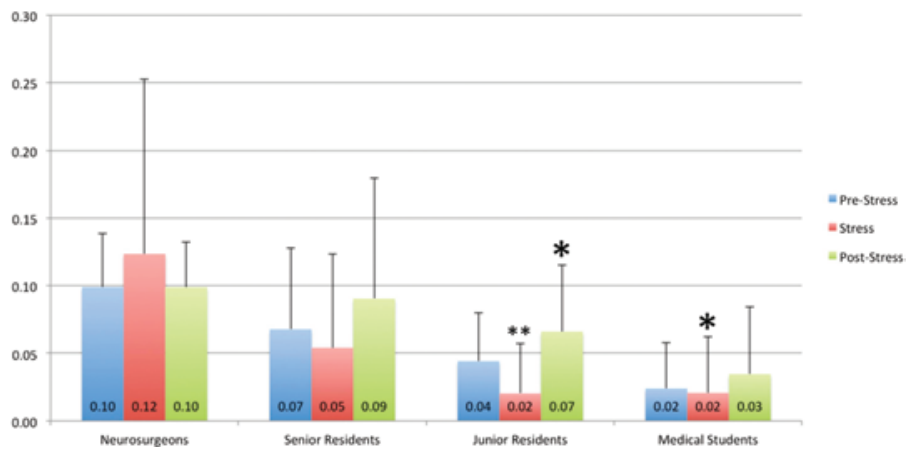

FIG. 2. Tier 1 metrics-blood loss $\left(\mathrm{cm}^{3}\right)$, tumor percentage resected, and brain volume removed $\left(\mathrm{cm}^{3}\right)$ - for the participant groups: neurosurgeon $(n=6)$, senior resident $(n=6)$, junior resident $(n=6)$, and medical student $(n=6)$. Statistical differences among baseline, stress, and post-stress values (B-D) are represented as ${ }^{*} p<0.05$ and ${ }^{* *} p<0.01$. Arrows $(\mathbf{A})$ indicate which groups were statistically different from other groups $(p<0.05)$ at baseline. Figure is available in color online only.

a statistical improvement in performance was seen. A significant decrease in the amount of blood loss was also seen in the senior resident group, as was a significant increase in the percentage of tumor resected. An increase in the Tier 1 metrics of tumor percentage resection and brain volume removed was the only statistically significant change seen in the junior resident group. No significant differences were seen in any of the metrics studied when pre- and post-stress performances were compared for the student group.

\section{Group Analysis}

The stress scenario was designed to prevent the participants from controlling bleeding and a significant increase in this Tier 1 metric was seen for all groups. Baseline blood loss was significantly lower and the percentage of tumor removed was significantly higher in the neurosurgeon group, consistent with their "expert" designation. A number of the baseline advanced Tier 2 metrics clearly differentiate the neurosurgeon group from the other groups, consistent with the validated metrics that we have previously reported. ${ }^{1,2}$ Regardless of the group assessed, the advanced Tier 2 metrics' bimanual psychomotor performance tasks were predominately the ones significantly altered during the stress scenario. In comparing pre-stress and immediate post-stress results, we noted that only Tier 1 metrics were increased, suggesting that the majority of the assessed metrics are not significantly changed by stress associated with simulated uncontrollable bleeding in this tumor resection scenario.

\section{Discussion}

The NeuroTouch platform with the multiple metrics we have validated allowed the measurement of bimanual psychomotor performance before, during, and after a stress scenario. ${ }^{1,2,8}$

\section{Differentiating Expert From Novice Performance During Acute Stress}

Neurosurgeon psychomotor performance during this acute stress scenario has multiple interactive aspects that can be dissected. Participants showed a statistically significant increase in their STAI scores when baseline and post-trial values were compared. Neurosurgeons had the highest mean values on the baseline STAI questionnaire, suggesting that participating in these simulated scenarios does cause them subjective stress. Interestingly neurosurgeons and senior residents were the only groups to state on the questionnaire that they did not experience more stress after the trial compared with before it. In a study by Sexton et al., surgeons, as compared with pilots, were more likely to deny the effect of fatigue on performance. ${ }^{30}$ This finding may explain comments we saw.

Figure 6 provides a summary heat map of significant results for Tier 1, Tier 2, and advanced Tier 2 metrics for each 
A

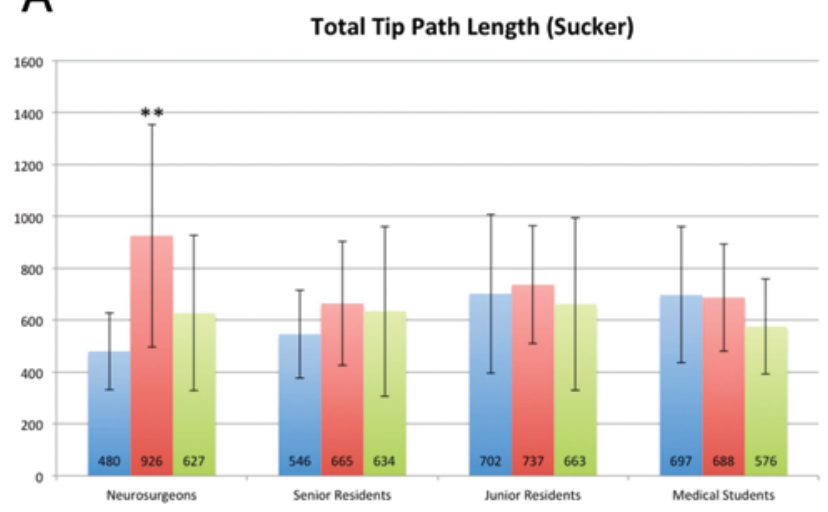

B

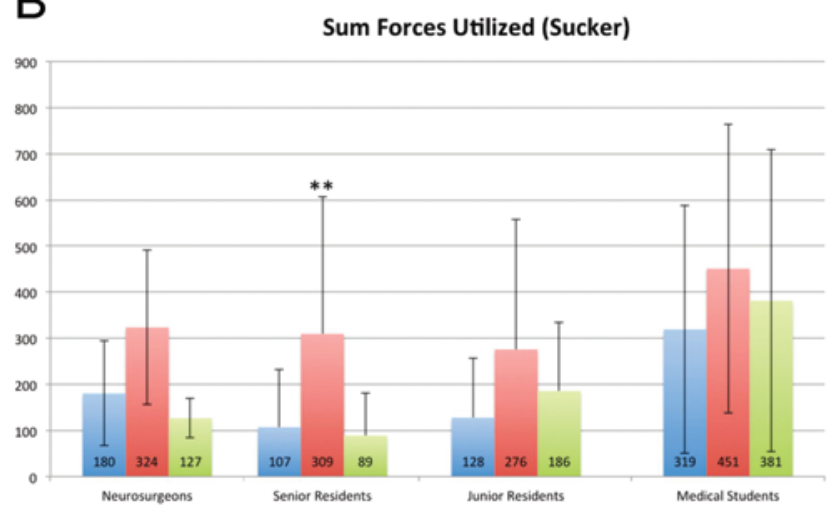

C

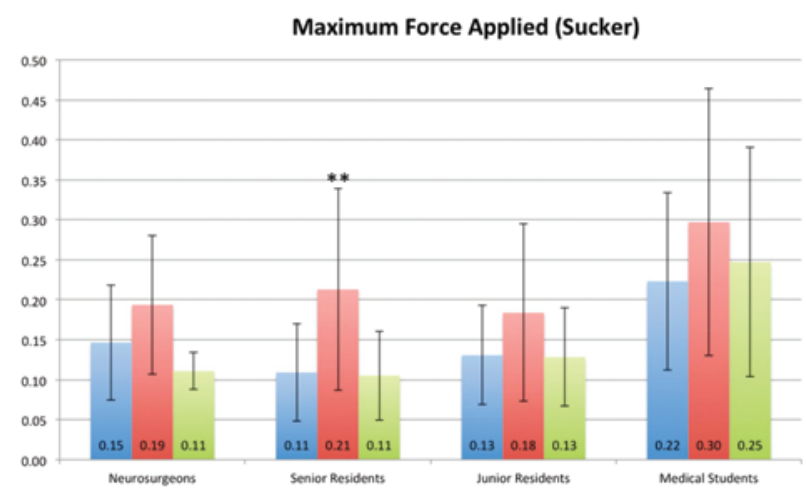

Total Tip Path Length (Aspirator)

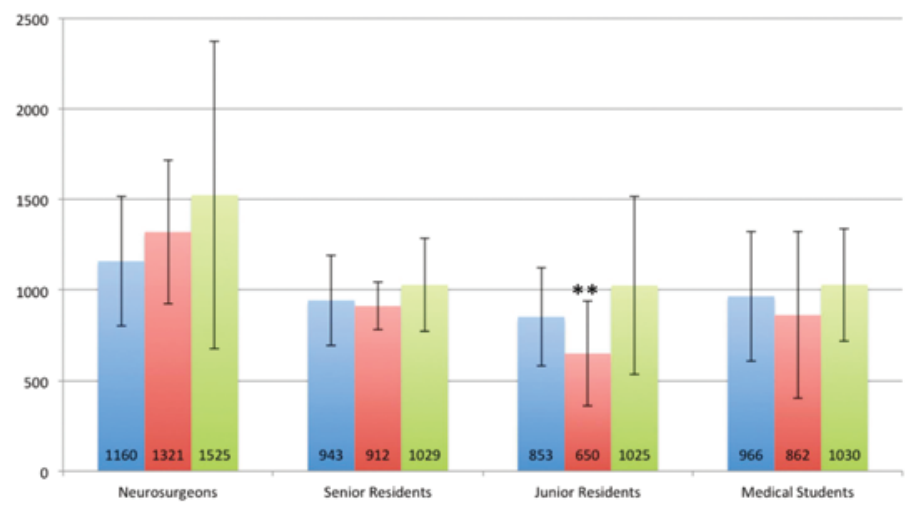

Sum Forces Utilized (Aspirator)

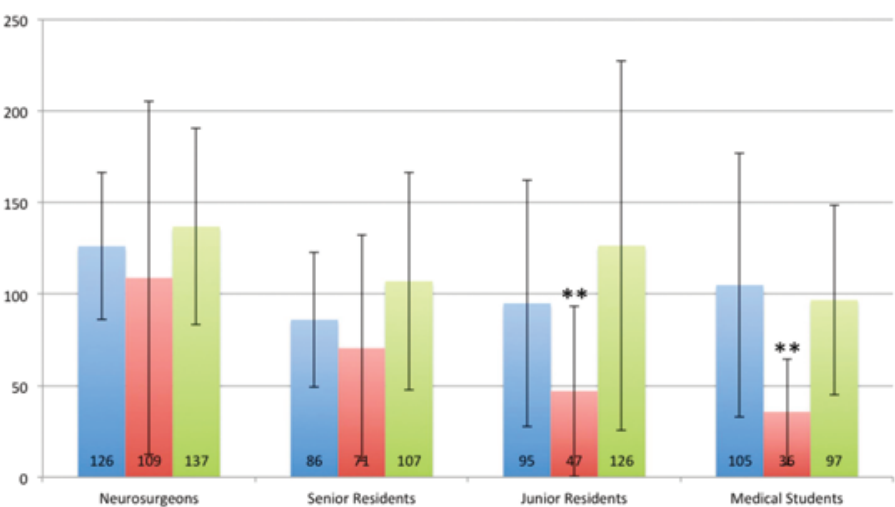

Maximum Force Applied (Aspirator)

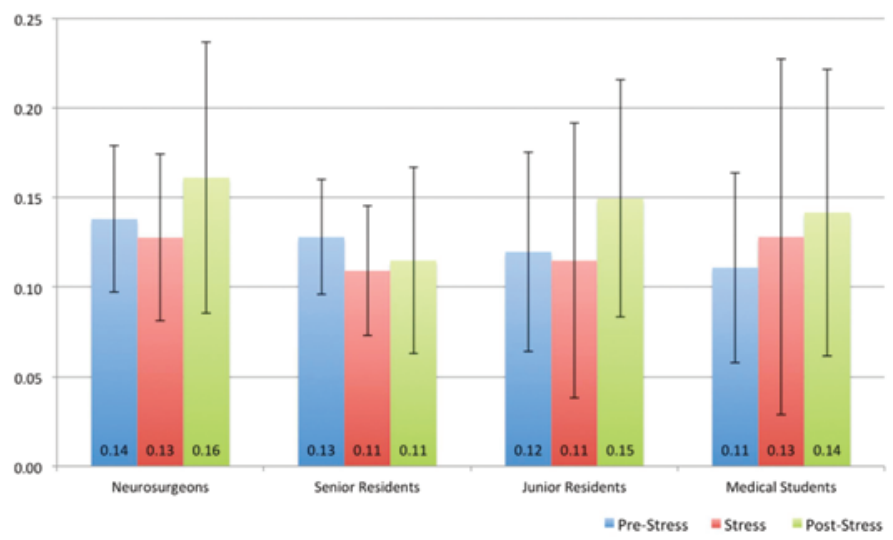

FIG. 3. Tier 2 metrics-total tip path length (mm), sum forces used (N), and maximum force applied (N)-of participant groups at baseline and during and immediately following the stress scenario. Statistical differences among baseline, stress, and post-stress values are represented as ${ }^{*} p<0.05$ and ${ }^{* *} p<0.01$. Figure is available in color online only.

group in comparing baseline and stress-scenario values. For Tier 1 metrics all groups have significantly increased blood loss as expected, but only the junior residents and students show significant decreases in the percentage tumor resected and brain volume removed that are associated with the bleeding. Neurosurgeon baseline mean percentage tumor resected was $94 \% \pm 6.9 \%$, while senior and junior resident values were $67 \% \pm 24.3 \%$ and $68 \% \pm 17.8 \%$, respectively. This mean percentage tumor resected by neurosurgeons decreased to $82 \% \pm 19.4 \%$ during the stress scenario, including 2 neurosurgeons who completely resected the tumor despite the bleeding; values for the senior and junior residents decreased as well to $54 \% \pm 28.8 \%$ and $36 \%$ $\pm 27.5 \%$, respectively. Although these decreases in resection performance values are somewhat equivalent, neurosurgeons performed better during the stress episode than the residents did at baseline. To improve resident performance during such bleeding episodes, more focus on their ability to conduct resections both before and during stress scenarios seems warranted.

For Tier 2 metrics the major significant differences are found between neurosurgeons and senior residents. Neu- 


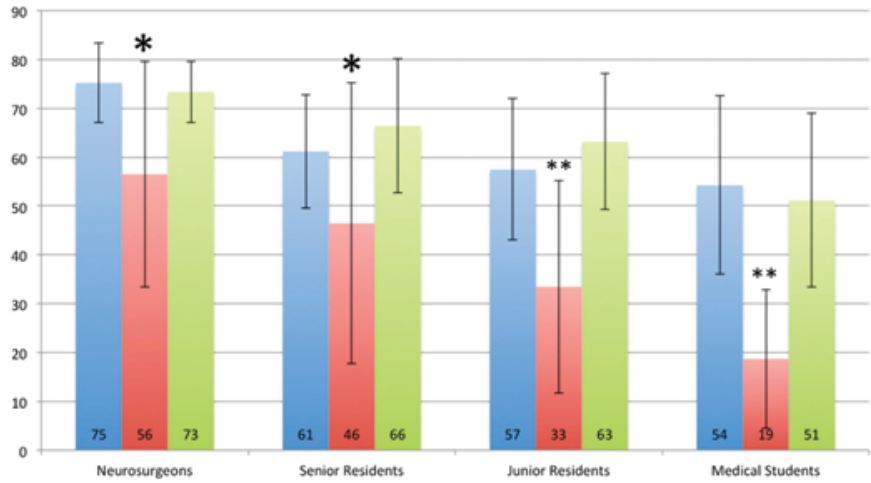

B

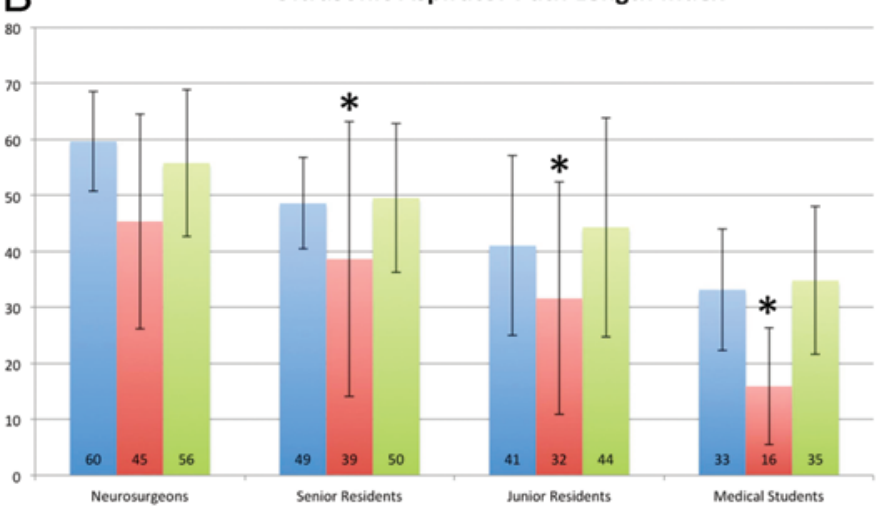

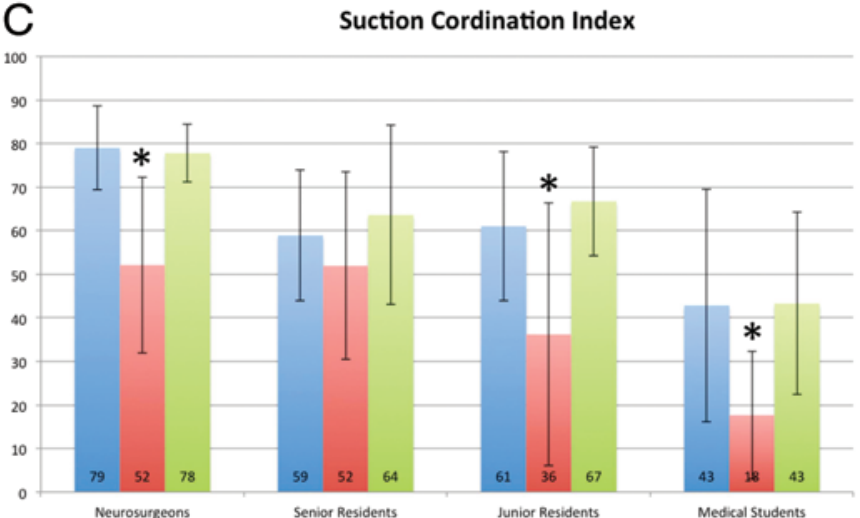

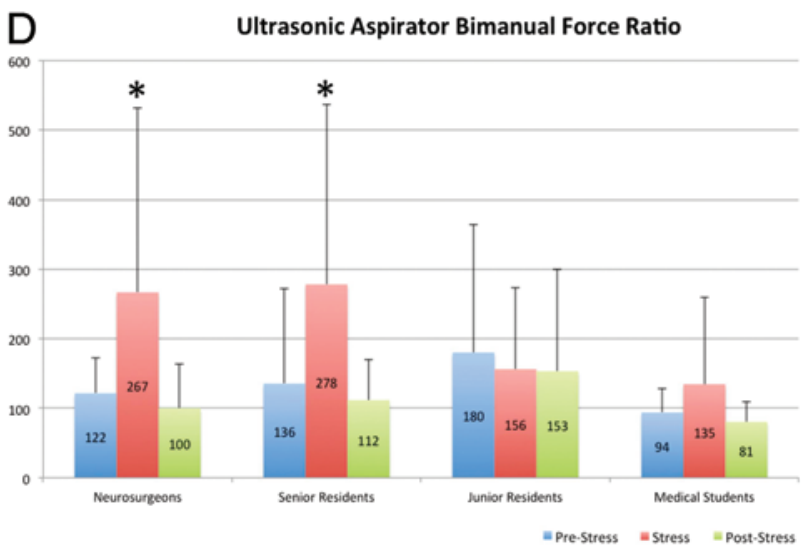

FIG. 4. Advanced Tier 2 metrics-efficiency index, suction coordination index, and ultrasonic path length index and bimanual force ratio-of participant groups at baseline and during and immediately following the stress scenario. Statistical differences among baseline, stress, and post-stress values are represented as ${ }^{*} p<0.05$ and ${ }^{* *} p<0.01$. Figure is available in color online only.

rosurgeons significantly increased the sucker total tip path length in an attempt to decrease blood loss, improve visibility, and resect tumor while not significantly increasing their sucker sum and maximum forces used. Senior residents, on the other hand, did not significantly increase the sucker total tip path length but did significantly increase the sucker sum and maximum forces used. If expert psychomotor behavior in this simulated bleeding scenario is equivalent to expert operating room performance, one might conclude that neurosurgeons have learned to increase sucker movement and control sucker forces during bleeding situations. These strategies improve visualization and tumor resection while mitigating the risk of excessive instrument force on brain tissues, which could impact patient safety. Using these insights, residents can train to increase sucker movement and modulate sucker forces when controlling bleeding while using simulators. Visual readouts of a resident's instrument movement and force application, as compared with expert performance benchmarks, could be continuously provided during simulated operative procedures. ${ }^{1,8}$ This information would allow the learner to immediately modify his or her use of the instrument during the simulated task. An instructor with this information could modify learner performance with more specific appropriate guidance. Junior residents use both significantly less aspirator total tip path length and sum of forces used, suggesting decreased maximization of aspirator utility during bleeding, an issue addressable with simulations.

Advanced Tier 2 metrics results help to explain several Tier 1 and 2 results. Neurosurgeons had significantly higher baseline efficiency, suction coordination, and ultrasonic aspirator path length indices, resulting in an ability to remove greater tumor volumes at baseline as well as during and after the stress event. The stress scenario was also associated with a significant increase in the ultrasonic aspirator bimanual force ratios index. This finding suggests that the coordinated use of the aspirator and sucker are important components of experts trying to deal with the bleeding. These activities are amenable to simulator training.

\section{Psychomotor Performance Following Acute Stress}

After experiencing an acute stress event, individuals can improve, worsen, or maintain psychomotor performance. ${ }^{6,11,23,25}$ Tumors 5 and 6 were specifically included to assess this issue. An alternate reason for improved performance involving a series of similar scenarios could be the learning effect of repetition. ${ }^{19}$ Significant improvements in neurosurgeon and senior resident bimanual psychomotor performance were seen in blood loss. Senior and junior residents had an improved percentage of tumor resected 


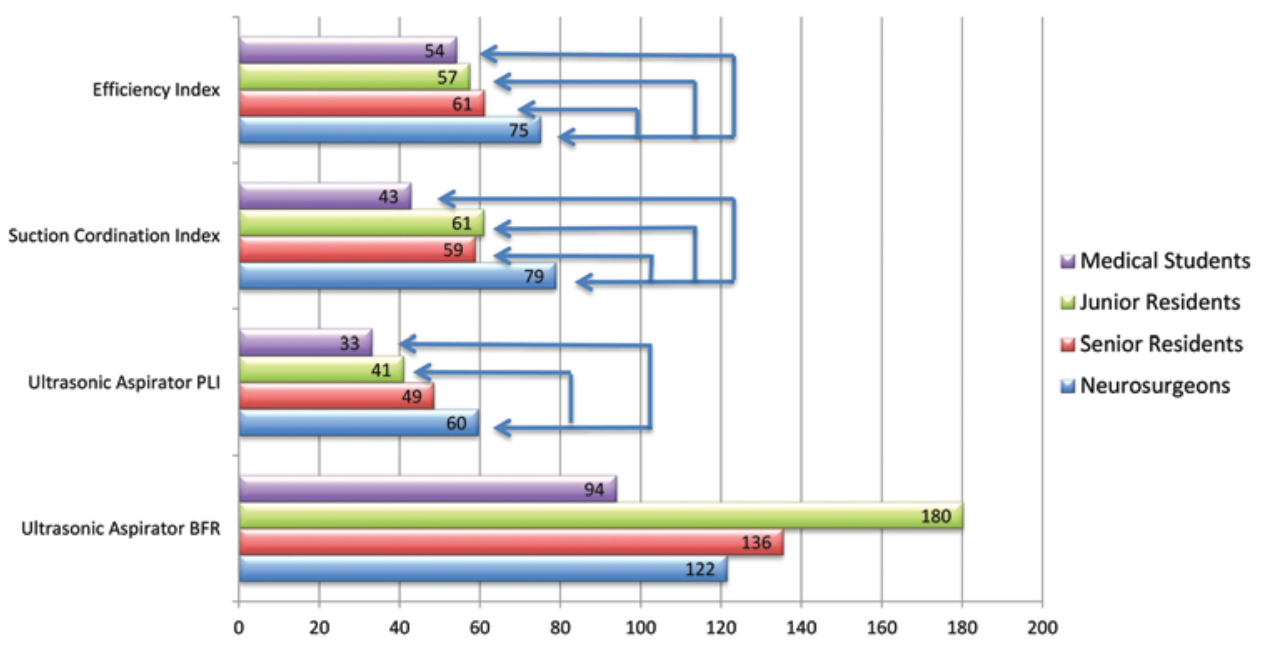

FIG. 5. Statistical differences in advanced Tier 2 metrics for the participant groups at baseline. Arrows indicate which groups are statistically different from other groups $(p<0.05)$. Figure is available in color online only.

after the acute stress episode, which was associated with significant increases in brain volume removed for the junior residents. Although it is not possible to eliminate a learning effect as the reason for these improvements since they occurred only in Tier 1 metrics and only in some groups, the results are more consistent with improved performance after stress. No psychomotor bimanual performance metrics demonstrated a statistically significant decrease in any group; therefore, this stress scenario is not associated with deteriorated bimanual psychomotor performance.

\section{Study Strengths and Limitations}

NeuroTouch technology has allowed us to address the 2 questions proposed for this study and has provided insights into how experts (neurosurgeons) and novices (residents) perform simulated brain tumor operations during and after a stress-inducing event. The baseline Tier 1 metrics of blood loss and total percentage of tumor resected differentiated the neurosurgeon and resident groups and provided construct validity for these metrics utilizing the NeuroTouch simulator. These metrics were not validated in 2 previous studies. ${ }^{1,3}$ The difference in these results may relate to different tumor structure, surrounding normal tissue, and resection sequence in the present study. Two of 3 baseline Tier 1 metrics differentiated neurosurgeon and resident groups. This finding corroborated our previous data indicating that for 3 of 4 advanced Tier 2 metrics, the major improvement in operative skills occurs following residency training. ${ }^{.}$Serial tracking of residents during training and after graduation is necessary to understand the psychomotor skills acquisition sequence during residency and the modification of these skills during neurosurgical practice. Guided by these validated metrics, we think it reasonable to propose the development of proficiency-based benchmarks for resident training curriculum and self-assessment programs to both assess and modulate stress during operative procedures. . $^{1,3,8,16}$

Caution is needed in the interpretation of our results. First, this study was focused on the effect of acute stress on psychomotor bimanual performance and should not be generalized to the influence of chronic stressors such as fatigue and other conditions. ${ }^{9,14,22}$ Second, operators were only allowed to use a simulated suction device and ultrasonic aspirator to control significant bleeding during the simulated tumor resection. These parameters are not representative of the multiple complex interactive skills and techniques available to address this problem during tumor resections in patients. The development of surgical judg-

\begin{tabular}{|c|c|c|c|c|}
\hline & Neurosurgeons & $\begin{array}{c}\text { Senior } \\
\text { Residents }\end{array}$ & $\begin{array}{c}\text { Junior } \\
\text { Residents }\end{array}$ & $\begin{array}{l}\text { Medical } \\
\text { Students }\end{array}$ \\
\hline \multicolumn{5}{|c|}{ Tier 1 Metrics } \\
\hline \multicolumn{5}{|l|}{ Blood Loss } \\
\hline \multicolumn{5}{|l|}{$\begin{array}{c}\text { Tumor Percentage } \\
\text { Resected }\end{array}$} \\
\hline \multicolumn{5}{|l|}{$\begin{array}{c}\text { Brain Volume } \\
\text { Removed }\end{array}$} \\
\hline \multicolumn{5}{|c|}{ Tier 2 Metrics } \\
\hline \multicolumn{5}{|l|}{$\begin{array}{l}\text { Total Tip Path Length } \\
\text { (Sucker) }\end{array}$} \\
\hline \multicolumn{5}{|l|}{$\begin{array}{l}\text { Sum Forces Utilized } \\
\text { (Sucker) }\end{array}$} \\
\hline \multicolumn{5}{|l|}{$\begin{array}{l}\text { Maximum Force } \\
\text { Applied (Sucker) }\end{array}$} \\
\hline \multicolumn{5}{|l|}{$\begin{array}{l}\text { Total Tip Path Length } \\
\text { (Aspirator) }\end{array}$} \\
\hline \multicolumn{5}{|l|}{$\begin{array}{l}\text { Sum Forces Utilized } \\
\text { (Aspirator) }\end{array}$} \\
\hline \multicolumn{5}{|l|}{$\begin{array}{l}\text { Maximum Force } \\
\text { Applied (Aspirator }\end{array}$} \\
\hline \multicolumn{5}{|c|}{ Advanced Tier 2 Metrics } \\
\hline \multicolumn{5}{|l|}{ Efficiency Index } \\
\hline \multicolumn{5}{|l|}{$\begin{array}{l}\text { Suction Coordination } \\
\text { Index }\end{array}$} \\
\hline \multicolumn{5}{|l|}{$\begin{array}{l}\text { Ultrasonic Aspirator } \\
\text { Path Length Index }\end{array}$} \\
\hline $\begin{array}{l}\text { Ultrasonic Aspirator } \\
\text { Bimanual Force Ratio }\end{array}$ & & & & \\
\hline
\end{tabular}

Red Color Indicate significant increase during stress, $\mathrm{P}<0.05$

Blue Color Indicate significant decrease during stress, $\mathrm{P}<0.05$

Gray Color Indicate no significant change during stress, $\mathrm{P}>0.05$

FIG. 6. Heat map of statistically significant affected metrics when baseline and stress-scenario values were compared $(p<0.05)$. Figure is available in color online only. 
ment and experience essential to preventing severe bleeding and dealing with intraoperative cardiac arrest is critical to resident learning, and the scenarios outlined in this trial do not address these important issues. Third, the task quality, difficulty, and short duration of the stress scenario may not adequately discriminate performance among such a limited number of operators studied $(n=24)$. These issues along with the type of metrics employed may have resulted in our inability to find more significant differences. These metrics are being assessed for their usefulness in other simulated neurosurgical operations and by other surgical specialties, which may aid the universality of their application. ${ }^{17,18,29,31,32}$ Fourth, the use of neurosurgeons, residents, and students from only 1 institution may have resulted in our inability to find more significant differences between groups. Fifth, the relationship of psychomotor performance to stress-induced physiological changes is important in the dissection of operator response to anxiety-inducing situations such as severe bleeding and will be addressed in further communications. ${ }^{13,33}$ This study was focused on the assessment of the influence of acute stress on bimanual psychomotor performance, but the utility of virtual reality simulators like NeuroTouch will be limited unless it can be shown that they enhance resident operating room performance..$^{8,16,21}$

\section{Conclusions}

Our study is the first to demonstrate that acute stress initiated by severe intraoperative bleeding during the resection of a simulated brain tumor significantly decreased bimanual psychomotor performance during the acute episode. The simulated intraoperative bleeding event had no significant influence on the Tier 2 and advanced Tier 2 metrics monitored during immediate post-stress operative performance but did significantly improve Tier 1 control of bleeding in the neurosurgeon and senior resident groups.

\section{Acknowledgments}

We thank Dr. Robert DiRaddo, group leader, Simulation, Life Sciences Division, National Research Council of Canada at Boucherville, and all his team, including Denis Laroche, Valérie Pazos, Nusrat Choudhury, Patricia Debergue, and Linda Pecora, for their support in developing the scenarios used in these studies and Dr. Roy Dudley, Department of Neurosurgery, McGill University, for his input. We also acknowledge the support of Drs. Mahmoud Al-Yamany and Lahbib Soualmi, National Neuroscience Institute, Department of Neurosurgery, King Fahad Medical City, Riyadh, and Dr. Saleh Baeesa, King Abdulaziz University, Jeddah, Saudi Arabia.

This work was supported by the Di Giovanni Foundation, the Montreal English School Board, the B-Strong Foundation, the Tony Colannino Foundation, and the Montreal Neurological Institute and Hospital.

\section{References}

1. Alotaibi FE, AlZhrani GA, Mullah MA, Sabbagh AJ, Azarnoush H, Winkler-Schwartz A, et al: Assessing bimanual performance in brain tumor resection with NeuroTouch, a virtual reality simulator. Neurosurgery 11 (Suppl 2):89-98, 2015

2. Alotaibi FE, AlZhrani GA, Sabbagh AJ, Azarnoush H, Win-
kler-Schwartz A, Del Maestro RF: Neurosurgical assessment of metrics including judgment and dexterity using the virtual reality simulator NeuroTouch (NAJD Metrics). Surg Innov 22:636-642, 2015

3. AlZhrani G, Alotaibi F, Azarnoush H, Winkler-Schwartz A, Sabbagh A, Bajunaid K, et al: Proficiency performance benchmarks for removal of simulated brain tumors using a virtual reality simulator NeuroTouch. J Surg Educ 72:685696, 2015

4. Arora S, Hull L, Sevdalis N, Tierney T, Nestel D, Woloshynowych M, et al: Factors compromising safety in surgery: stressful events in the operating room. Am J Surg 199:6065,2010

5. Arora S, Sevdalis N, Nestel D, Tierney T, Woloshynowych M, Kneebone R: Managing intraoperative stress: what do surgeons want from a crisis training program? Am J Surg 197:537-543, 2009

6. Arora S, Sevdalis N, Nestel D, Woloshynowych M, Darzi A, Kneebone R: The impact of stress on surgical performance: a systematic review of the literature. Surgery 147:318-330, 330 e311-316, 2010

7. Arora S, Tierney T, Sevdalis N, Aggarwal R, Nestel D, Woloshynowych M, et al: The Imperial Stress Assessment Tool (ISAT): a feasible, reliable and valid approach to measuring stress in the operating room. World J Surg 34:1756-1763, 2010

8. Azarnoush H, Alzhrani G, Winkler-Schwartz A, Alotaibi F, Gelinas-Phaneuf N, Pazos V, et al: Neurosurgical virtual reality simulation metrics to assess psychomotor skills during brain tumor resection. Int J Comput Assist Radiol Surg 10:603-618, 2015

9. Balch CM, Freischlag JA, Shanafelt TD: Stress and burnout among surgeons: understanding and managing the syndrome and avoiding the adverse consequences. Arch Surg 144:371376, 2009

10. Choudhury N, Gélinas-Phaneuf N, Delorme S, Del Maestro R: Fundamentals of neurosurgery: virtual reality tasks for training and evaluation of technical skills. World Neurosurg 80:e9-e19, 2013

11. Davis P, Sime WE, Robertson J: Sport psychophysiology and peak performance applications of stress management, in Lehrer PM, Woolfolk RL, Sime WE (eds): Principles and Practice of Stress Management, ed 3. New York: Guilford Press, 2007, pp 615-637

12. Delorme S, Laroche D, DiRaddo R, Del Maestro RF: NeuroTouch: a physics-based virtual simulator for cranial microneurosurgery training. Neurosurgery 71 (1 Suppl Operative):32-42, 2012

13. Dupee M, Werthner P: Managing the stress response: The use of biofeedback and neurofeedback with Olympic athletes. Biofeedback 39:92-94, 2011

14. Ganju A, Kahol K, Lee P, Simonian N, Quinn SJ, Ferrara JJ, et al: The effect of call on neurosurgery residents' skills: implications for policy regarding resident call periods. J Neurosurg 116:478-482, 2012

15. Gélinas-Phaneuf N, Choudhury N, Al-Habib AR, Cabral A, Nadeau E, Mora V, et al: Assessing performance in brain tumor resection using a novel virtual reality simulator. Int $\mathbf{J}$ CARS 9:1-9, 2014

16. Gélinas-Phaneuf N, Del Maestro RF: Surgical expertise in neurosurgery: integrating theory into practice. Neurosurgery 73 (Suppl 1):30-38, 2013

17. Gould D, Maynard I: Psychological preparation for the Olympic Games. J Sports Sci 27:1393-1408, 2009

18. Holloway T, Lorsch ZS, Chary MA, Sobotka S, Moore MM, Costa AB, et al: Operator experience determines performance in a simulated computer-based brain tumor resection task. Int J CARS 10:1853-1862, 2015

19. Hopper AN, Jamison MH, Lewis WG: Learning curves in surgical practice. Postgrad Med J 83:777-779, 2007 
20. Laird NM, Ware JH: Random-effects models for longitudinal data. Biometrics 38:963-974, 1982

21. Kirkman MA, Ahmed M, Albert AF, Wilson MH, Nandi D, Sevdalis N: The use of simulation in neurosurgical education and training. J Neurosurg 121:228-246, 2014

22. Lazarus R, Folkman S: Cognitive theories of stress and the issue of circularity, in Appley MH, Trumbull RA (eds): Dynamics of Stress. New York: Springer, 1986, pp 63-80

23. LeBlanc VR: The effects of acute stress on performance: implications for health professions education. Acad Med 84 (10 Suppl):S25-S33, 2009

24. Marteau TM, Bekker H: The development of a six-item shortform of the state scale of the Spielberger State-Trait Anxiety Inventory (STAI). Br J Clin Psychol 31:301-306, 1992

25. McEwen BS, Wingfield JC: The concept of allostasis in biology and biomedicine. Horm Behav 43:2-15, 2003

26. Müller M: Safety lessons taken from the airlines. Br J Surg 91:393-394, 2004

27. Novaco R, Cook T, Sarason I: Military recruit training: an arena for stress-coping skills, in Jaremko M, Meichenbaum D (eds): Stress Reduction and Prevention. New York: Springer, 1989, pp 377-418

28. Ros T, Moseley MJ, Bloom PA, Benjamin L, Parkinson LA, Gruzelier JH: Optimizing microsurgical skills with EEG neurofeedback. BMC Neurosci 10:87, 2009

29. Rosseau G, Bailes J, del Maestro R, Cabral A, Choudhury N, Comas O, et al: The development of a virtual simulator for training neurosurgeons to perform and perfect endoscopic endonasal transsphenoidal surgery. Neurosurgery 73 (Suppl 1):85-93, 2013

30. Sexton JB, Thomas EJ, Helmreich RL: Error, stress, and teamwork in medicine and aviation: cross sectional surveys. BMJ 320:745-749, 2000

31. Varshney R, Frenkiel S, Nguyen LH, Young M, Del Maestro $\mathrm{R}$, Zeitouni A, et al: Development of the McGill simulator for endoscopic sinus surgery: a new high-fidelity virtual reality simulator for endoscopic sinus surgery. Am J Rhinol Allergy 28:330-334, 2014

32. Varshney R, Frenkiel S, Nguyen LH, Young M, Del Maestro
R, Zeitouni A, et al: The McGill simulator for endoscopic sinus surgery (MSESS): a validation study. J Otolaryngol Head Neck Surg 43:40, 2014

33. Werthner P, Christie S, Dupee M: Neurofeedback and biofeedback training with Olympic athletes. NeuroConnections (Summer):32-37, 2013

34. Wetzel CM, Kneebone RL, Woloshynowych M, Nestel D, Moorthy K, Kidd J, et al: The effects of stress on surgical performance. Am J Surg 191:5-10, 2006

\section{Disclosures}

The authors report no conflict of interest concerning the materials and methods in this study or the findings specified in this paper.

\section{Author Contributions}

Conception and design: Bajunaid, Alotaibi, Fares, Baggiani, Azarnoush, Christie, Al-Zhrani, Werthner, Del Maestro. Acquisition of data: Alotaibi, Fares, Baggiani, Azarnoush, Christie, AlZhrani, Sabbagh, Werthner, Del Maestro. Analysis and interpretation of data: Bajunaid, Winkler-Schwartz, Alotaibi, Marwa, Del Maestro. Drafting the article: Bajunaid, Winkler-Schwartz. Critically revising the article: Bajunaid, Mullah, Winkler-Schwartz, Christie, Del Maestro. Reviewed submitted version of manuscript: all authors. Approved the final version of the manuscript on behalf of all authors: Bajunaid. Statistical analysis: Mullah. Study supervision: Del Maestro.

\section{Supplemental Information Videos \\ Video 1. https://vimeo.com/150258112.}

\section{Correspondence}

Khalid Bajunaid, Neurosurgical Simulation Research Centre, Department of Neurosurgery, Montreal Neurological Institute and Hospital, McGill University, 3801 University St., Rm. W201, Montreal, QC H3A 2B4, Canada. email: khalid.bajunaid@mail. mcgill.ca. 